INPLASY

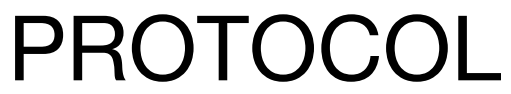

To cite: Luo et al. The add-on effect of Chinese herbal medicine on COVID-19: a systematic review and metaanalysis. Inplasy protocol 202040190. doi:

10.37766/inplasy2020.4.0190

Received: 27 April 2020

Published: 27 April 2020

Corresponding author:

Xiaojia Ni

grace1984325@126.com

Author Affiliation:

Guangdong Provincial Hospital

of Chinese Medicine

Support: GPKLRETCM

(No.2017B030314176)

Review Stage at time of this submission: Risk of bias assessment.

Conflicts of interest: None.

\section{The add-on effect of Chinese herbal medicine on COVID-19: a systematic review and meta-analysis}

Luo, X1; Ni, X2; Lin, J3; Zhang, Y4; Wu, L5; Huang, D6; Liu, Y7; Cai, Y8; Chen, $\mathrm{Y}^{9}$; Lin L10.

Review question / Objective: Is the adjunct use of Chinese herbal medicine to conventional medication safely improve the patients with COVID-19?

Condition being studied: In December 2019, unexplained pneumonia emerged in Wuhan, China, and has since then spread rapidly throughout the country. The disease is caused by SARS-CoV-2, a new type of coronavirus, and it usually presents manifestations of pneumonia, such as cough, fever, fatigue, and dyspnea. On 11 February 2020, the World Health Organization (WHO) named the disease caused by the virus as COVID-19. On 30 January 2020, the WHO declared the outbreak a Public Health Emergency of International Concern (PHEIC)and on 11 March 2020, as pandemic. As of 15 April 2020, more than 1,910,000 cases had been diagnosed in 210 countries outside China, over 123,000 deaths by this date.

INPLASY registration number: This protocol was registered with the International Platform of Registered Systematic Review and Meta-Analysis Protocols (INPLASY) on 27 April 2020 and was last updated on 27 April 2020 (registration number INPLASY202040190).

\section{INTRODUCTION}

Review question / Objective: Is the adjunct use of Chinese herbal medicine to conventional medication safely improve the patients with COVID-19?

Condition being studied: In December 2019, unexplained pneumonia emerged in
Wuhan, China, and has since then spread rapidly throughout the country. The disease is caused by SARS-CoV-2, a new type of coronavirus, and it usually presents manifestations of pneumonia, such as cough, fever, fatigue, and dyspnea. On 11 February 2020, the World Health Organization (WHO) named the disease caused by the virus as COVID-19. On 30 
January 2020, the WHO declared the outbreak a Public Health Emergency of International Concern (PHEIC)and on 11 March 2020, as pandemic. As of 15 April 2020 , more than $1,910,000$ cases had been diagnosed in 210 countries outside China, over 123,000 deaths by this date.

Rationale: In China, Chinese authorities attached great importance to the use of Chinese herbal medicine (CHM) during the fight against the COVID-19 epidemic. However, there was not a comprehensive systematic review assessing the direct clinical evidence on Chinese herbal medicine for COVID-19 in terms of effectiveness and safety.

\section{METHODS}

Participant or population: The patients with COVID-19.

Intervention: Chinese herbal medicine in addition to conventional medicine.

Comparator: Conventional medicine with or without placebo.

Study designs to be included: Controlled clinical trial.

Eligibility criteria: We included studies if they meet the following criteria: 1) The participants was diagnosed as COVID-19 according to Chinese National Health Commission; 2) The intervention was CHM in addition to conventional medicine; 3 ) The control included conventional with or without placebo; and 4) Study types were controlled clinical trials.

Information sources: We searched the CNKI, CBM, Wanfang Data, PubMed, Cochrane Library, Embase, and Chinese Journal Medical Network (http:// medjournals.cn/2019NCP/index.do) form the inception to April 15, 2020. We also supplemented the search with Google Scholar, the journal preprint services that include ChemRxiv (https://chemrxiv.org/), medRxiv (https://www.medrxiv.org/), BioRxiv (https://www.biorxiv.org/) and SSRN (https://www.ssrn.com/index.cfm/ en/). Besides, references to the included literature were examined to supplement the included omitted literature, and studies included in published systematic reviews were also checked.

Main outcome(s): 1) Primary outcome: overall clinical effectiveness; 2) Secondary outcomes: the improvement in terms of CT scan, negative respondent rate of nucleic acid in respirotary specimens, the length of hospital stays, mortality, the disappearance rate of symptoms including cough, fatigue and fever, and adverse events.

Quality assessment / Risk of bias analysis: For randomised controlled trials, Cochrane risk of bias assessment was used; and for other controlled trials, New Castle-Ottawa Scale was used.

Strategy of data synthesis: We conducted meta-analyses by using STATA version $\mathbf{1 4 . 0}$ software. For dichotomous data, we calculated odds ratios (OR) with $95 \%$ confidence intervals (CI); for continuous data, we calculated weighted mean differences (WMD) with $95 \% \mathrm{Cl}$. Missing data were dealt with according to the Cochrane Handbook for Systematic Reviews of Interventions. Two-sided $\mathbf{P}$ values $50 \%$ indicating substantial heterogeneity.

Subgroup analysis: If we detected heterogeneity, we performed subgroup analyses by disease severity, study type, and complications.

Sensibility analysis: Sensitivity analysis was conducted by excluding outliers.

Language: Chinese and English.

Country(ies) involved: China.

Keywords: Chinese herbal medicine; COVID-19; Systematic review.

Contributions of each author:

Author 1 - Xufei Luo - Mr Xufei Luo conducted data-analysis and drafted the manuscript. 
Author 2 - Xiaojia Ni - Dr. Xiaojia Ni designed the study, interpreted the results, and drafted the manuscript.

Author 3 - Jiahui Lin - Mr Jiahui Lin searched the literature, collected the data and assessed the methodological quality of included studies.

Author 4 - Yidan Zhang - Miss Yidan Zhang searched the literature, collected the data, and assessed the methodological quality of the included studies.

Author 5 - Lei Wu - Dr Lei Wu assisted with the design of PICOs and interpreted the results.

Author 6 - Donghui Huang - Dr Donghui Huang assisted with the design of PICOs and interpreted the results.

Author 7 - Yuntao Liu - Dr Yuntao Liu assisted with the design of PICOs and interpreted the results.

Author 8 - Yefeng Cai - Professor Yefeng Cai conceived the study and oversought the study implementation.

Author 9 - Yaolong Chen - Professor Yaolong Chen provided the methodological guidance.

Author 10 - Lin Lin - Professor Lin Lin conceived the study, designed the PICOs and interpreted the study results. 